\title{
A Novel Fault Self-diagnosis Algorithm Based on Finite-state Machine for Space Information Network
}

\author{
Yuan Jiang, Ning Li, Fapeng Wang, Cong Wang and Lianguo Wu \\ Institute of Communication Engineering, PLA University of Science and Technology, Nanjing, China
}

\begin{abstract}
- the possibility of the development of space information network (SIN) was discussed in this paper, and a novel fault selfdiagnosis method was developed. There is limited research of space information network, especially in fault diagnosis domain. This paper gives expectation about future SIN features, and based on these features proposes a fault self-diagnosis algorithm based on finite-state machine. We design a series of novel fault detectors through which multiple satellites nodes can cooperate with each other in a diagnosis task. The fault detectors encode the diagnosis process to state transitions. Each satellites can participate in the fault diagnosis by transiting the detector's current state to a new one based on local evidence and then pass the fault detector to other satellites.
\end{abstract}

Keywords-self-diagnosis; space information network (SIN); finite-state machine

\section{INTRODUCTION}

Space information networks (SIN) [1] [2] were proposed to broaden the observation area and realize continuous information acquisition using satellites and high altitude platform stations [3]. SIN is able to enhance detection and transmission capabilities compared to the current single earth observation satellite. Unlike wireless sensor network, SIN is still a new concept since until now we cannot give even a particular explanation about it. Generally speaking, SIN is a heterogeneous type of network system, which takes satellite network as the backbone, and contains a variety of aircrafts, satellites, ground stations and terminals with space communication capability [4]. Since SIN is a newly emerging network style, the research about it is very limited especially in fault diagnosis domain. With its large coverage area, flexible networking, not being restricted by environment and other characteristics [5], SIN's fault diagnosis is more different than WSN. Paper [6] proposes a new routing strategy based on packet loss distinction. Routing maintenance strategy distinguishes the reasons of packet loss by sending high and low priority packets alternately. Then routing maintenance mechanisms are designed specifically according to different reasons. Paper [7] proposes a distributed cluster-based and fault-tolerant topology control algorithm to construct a more efficient and failure-resistant topology for space information networks. Paper [8] proposes a clustering algorithm. The proposed algorithm introduces a decision model based on Analytic hierarchy process (AHP) to select cluster heads, and then forms non-overlapping k-hop clusters. The dynamical selfmaintenance mechanisms take node mobility and cluster equalization into account. Besides of cluster merger/partition disposal, affiliation management and adaptive adjustment of information update period, mobile agents are used to migrate and duplicate functions of cluster heads in a recruiting way.

However, since the development of SIN, the network topology becomes more complexity. In 2013, NASA establishes Small Spacecraft Technology Program (SSTP) in response to the rapid growth in interest in using small spacecraft for missions beyond LEO [9]. Therefore, except existing satellites, thousands of small spacecraft is joining. As a result, subsistent fault diagnosis algorithm seems out of requirement. In this paper, considering about the small spacecraft's appearance we introduce a novel SIN system model and give the analysis about the system. And based on this model we propose an improved fault self-diagnosis algorithm [10] [11]. Small space craft will disappear when they fulfill their tasks. While in SIN system's point of view, it will lead to an inaccurate conclusion. As a result, in our improved algorithm first we need to judge whether it is in its working period or not. If it is out of its working period, we do not consider it as a fault. When it is in its working period, we run the main self-diagnosis algorithm. We design a series of novel fault detectors through which multiple satellites nodes can cooperate with each other in a diagnosis task. The fault detectors encode the diagnosis process to state transitions. Each satellite can participate in the fault diagnosis by transiting the detector's current state to a new one based on local evidence and then pass the fault detector to other satellites until it reaches at acceptable state. Consequently, we can finally get the wanted result. What's more, by the simulation result we can know that it performs well in space information network.

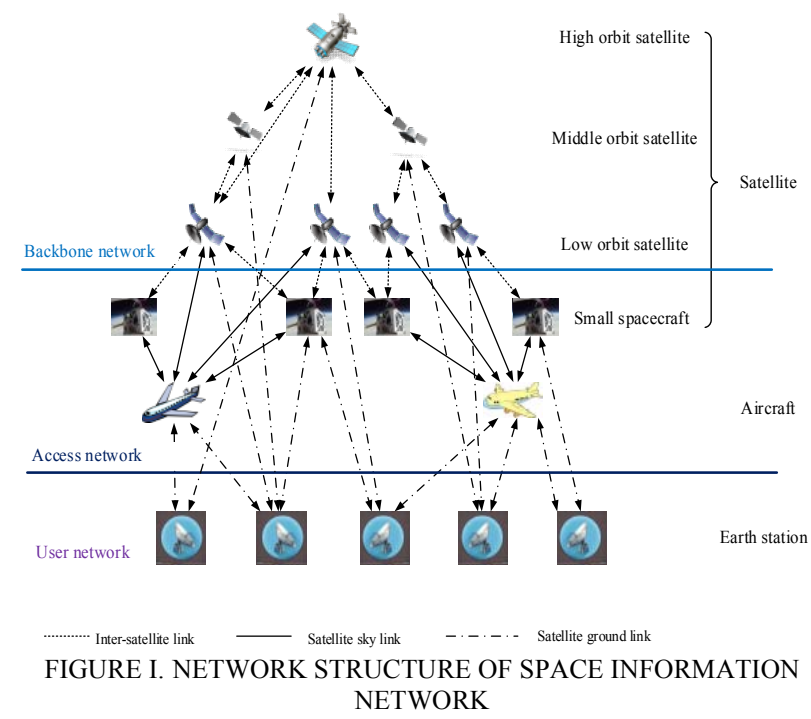


The rest of this paper is organized as follows. Section II introduces the novel system model and gives detailed analysis of it. Section III describes the fault detectors and the selfdiagnosis process. We discuss the implementation simulation result in Section IV. Finally, we conclude the work in Section $\mathrm{V}$.

\section{SYSTEM MODEL}

Figure 1 demonstrates the network model of SIN. It is obvious that it's a layered structure [12] [13] which can divide as backbone network, access network and user network. Backbone network combined with high, middle and low orbit satellites is the core of SIN system. It is responsible to gathering, transferring and rough processing information. Access network consisting of small spacecraft and aircraft is in charge of different access terminals and business flow's access. User domain is comprised of the every user terminals of space information network. As the development of small spacecraft technology, we can imagine thousands of small spacecraft will emerge in future days. Therefore, we need to make some adjustment in existing model. And compared with available SIN network model, since the joining of small spacecraft there are some new characteristics listed as follows.

\section{A. Small Spacecraft}

NASA gives a report in 2014 which names "Small Spacecraft Technology State of the Art". This report provides a summary of the state of the art for each of the following small spacecraft technology domains: Complete spacecraft, Power, Propulsion, Attitude Determination and Control, Structures, Materials and Mechanisms, Thermal Control, Command and Data Handling, Communications, Integration, Launch and Deployment, and Ground Data Systems and Operations. The majority of small spacecraft missions have a primary objective to collect scientific data and to transmit that data back to researchers on Earth. Therefore, small spacecraft's joining highly improves the QoS of the network.

\section{B. Characteristic}

\section{1) Complexity}

SIN ranges from the earth to the space, as everyone can image, there are various communication users and various network systems those users composing of, let alone those users also contains all kinds of satellites. Those infrastructures cooperate together into an efficient large-scare network system. For these reasons, unlike all the existed network system, the complexity of SIN can be imaginable. Therefore, the fault diagnosis of SIN system becomes much more difficult than any other network system existed.

\section{2) Dynamic and arbitrary}

For the joining of satellites and air crafts, the topology of network changes seriously. Location of nodes moves quickly as time going by. What's more, every node moves in its own law and their tracks overlaps in sometimes. The mobility of nodes also adds the arbitrary of the network system. And sometimes link between satellites disappears but next time the link appears again for satellites' location's changing. Worse still, small spacecraft is period working satellite. It means that once it fulfills its mission it will disappear immediately.

\section{FAUlt SELF-Diagonosis Algorithm}

Figure 2 give the overview of the proposed algorithm flow chat. As mentioned before, in order to deal with small spacecraft's short working period we design this algorithm. Assume that A detects an abnormal retransmission ratio change on its link $\mathrm{A} \rightarrow \mathrm{B}$. At the very beginning, we should make a judgment whether the selected node is in his working period or not. If it is out of its working period, we directly take it as a disappeared node return "no error" and update the topology. Else if it is in its working period then we can start our main algorithm. Detail information is listed as follows.

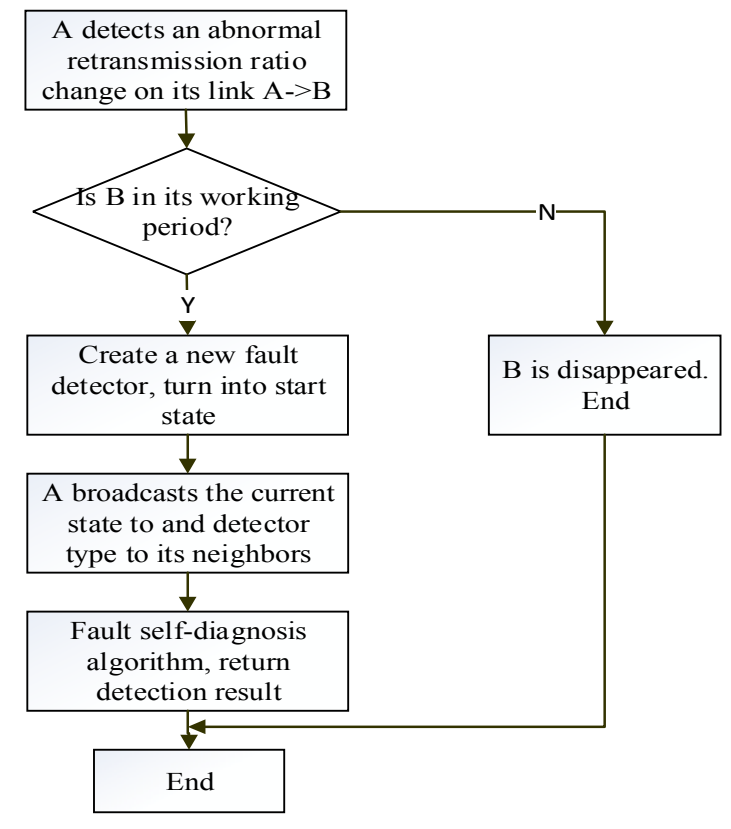

FIGURE II. SELF-DIAGNOSIS ALGORITHM FLOW CHAT

Figure 3 shows the finite-state machine in this paper. In figure 3 (a), we illustrate a partial network topology in which node $A$ is transmitting packets to node $B,\left\{C_{i}\right\}$ denotes a group of nodes in the neighboring region of $A$ and $B$ that can communicate with $A$ and $B$. $A$ detects that the retransmission ratio on link $A-->B$ abruptly turns to be significantly high, so $A$ creates a new fault detector as shown in figure 3 (b). Formally, the fault detector $M$ is represented as a quintuple $M=$ $\left(E, S, S_{0}, f, F\right)$ where $E$ denotes the set of input evidences, $S$ is the set of states in which $S_{0}$ is called the Start state, $F$ denotes a subset of $S$ that includes all Accept states, $f$ are the set of statetransition functions which specify the conditions for state switching. In the example of figure $3(\mathrm{~b})$, cyclic vertices $S_{i}$ denote states in $S$, each arc indicates a possible transition from one state to another and annotations on the arcs specify the transition conditions. Note that some states may have self-loops. States denoted by double cycles are the Accept states which represent final diagnosis decisions. When a fault detector reaches an Accept state, we can conclude the fault type and root causes. 


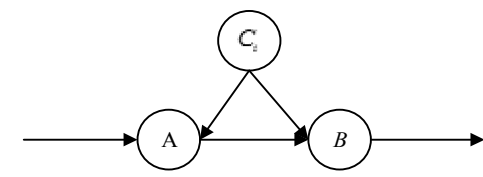

(a)

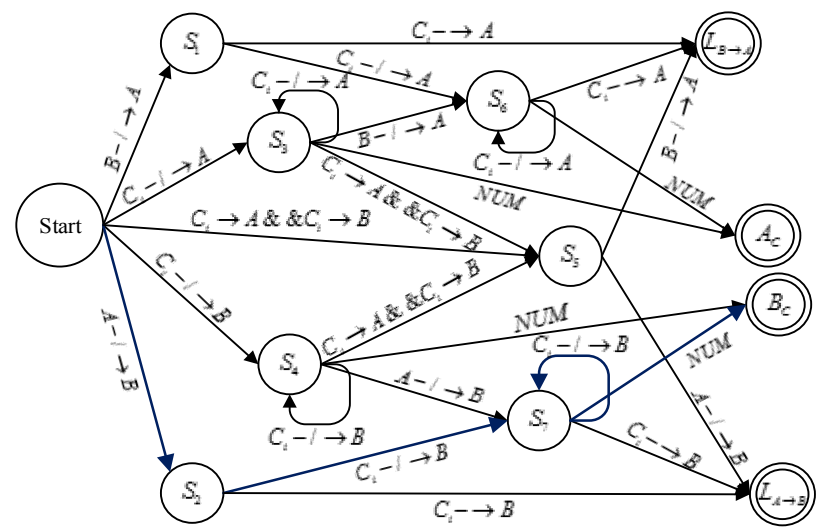

(b)

FIGURE III. (a) A PATIAL NETWORK TOPOLOGY. (b) THE DATA STRUCTURE FAULT DETECTOR

Now we describe the details of a diagnosis process with the detector shown in figure 3 (b). $A$ creates a new fault detector which is now at the Start state. In this detector, the Start state has the meaning that some node $A$ finds that its transmission performance experiences problems on link $A-->B$. $A$ then broadcasts the current state together with the detector type to its neighbors. Two categories of nodes may receive and handle this state, the first category includes the receiver $B$ and the second category contains a group of nodes that have recently transmits packets to $A$ or $B$ denoted as $\left\{C_{i}\right\}$.

Based on their local knowledge, they can make different transitions. For example, if $B$ receives the Start state, it checks its local evidences for the fact that if it has just received duplicated data packets from $A$. Since recording all detailed acknowledgment information is costly, here the duplicate reception is used as an indication of whether $B$ has received and acknowledged the data packets from $A$. If $B$ has acknowledged $A$ but still receives the same data packet from $A$, it means that $A$ successfully sends the packets to $B$ but fails to receive the acknowledgment from $B$, otherwise it means that $B$ has difficulties in receiving data packets from $A$. The arcs with conditions $B-/->A$ and $A-/->B$ represent the two situations, respectively. Based on $B$ 's local evidences, we can change the state from Start to $S_{1}$ or $S_{2}$. In this example, as illustrated by the blue arc in Fig. 3(b), $B$ does not receive enough data packets from $A$, so $B$ transits the state to $S_{2}$ and rebroadcast this state. $S_{2}$ can be handled by nodes in $\left\{C_{i}\right\}$. If the local information of $C_{i}$ shows that the data delivery from $C_{i}$ to $B$ is successful, it can be inferred that the poor link quality on link $A-->B$ leads to the high packet loss (frequent retransmissions). In this case, $C_{i}$ transits the detector state to an Accept state $L_{A->B}$ which indicates a poor link quality on link $A-->B$. Otherwise, if $C_{i}$ can hardly transmit data packets to $B$ as well, the state is transited to $S_{7}$ as shown by the blue arc. Note that the state $S_{7}$ has self-loops on condition $C_{i}-/->B$. Take $S_{7}$ for example, if more than a certain number (threshold) of nodes say that they have problems in communicating with $B\left(C_{i}-/->B\right)$, we transit the state to the Accept state $B_{c}$ which indicates that there are severe contention at $B$ and node $B$ is probably congested. Obviously, for the existence of threshold it may mislead to correct result. But it is worth to decrease the complexity of the algorithm.

\section{SIMULATION}

In this section, we give some simulation information and result to prove the proposed algorithm. Our simulation tool is MATLAB2010. In order to make this judgment we set 6 elements of every node's structure, $X=\left\{L_{A}, L_{B}, T, t, R_{A}, R_{B}\right\} . L_{A}$ represent the link relationship with $A$ at that moment. Similarly, $L_{B}$ represent the link relationship with $B . T$ means the working period of the node, $t$ stands for the satellite nodes' runs on orbit. $R_{A}, \quad R_{B}$ means the topology relationship with $A$ and $B$ accordingly. Therefore, we can run our algorithm.

TABLE I. OVERVIEW OF DIRECTED GRAPH DATASETS OF THRESHOLD

\begin{tabular}{|c|c|}
\hline Number of nodes & 355 \\
\hline Number of geostationary satellite nodes & 3 \\
\hline Number of middle orbit satellite nodes & 18 \\
\hline Number of low orbit satellite nodes & 72 \\
\hline Number of spacecraft nodes & 150 \\
\hline Number of air craft nodes & 100 \\
\hline Number of base station nodes & 10 \\
\hline Circulate number $\boldsymbol{i}$ & $2,3,4,5,6,7$ \\
\hline
\end{tabular}

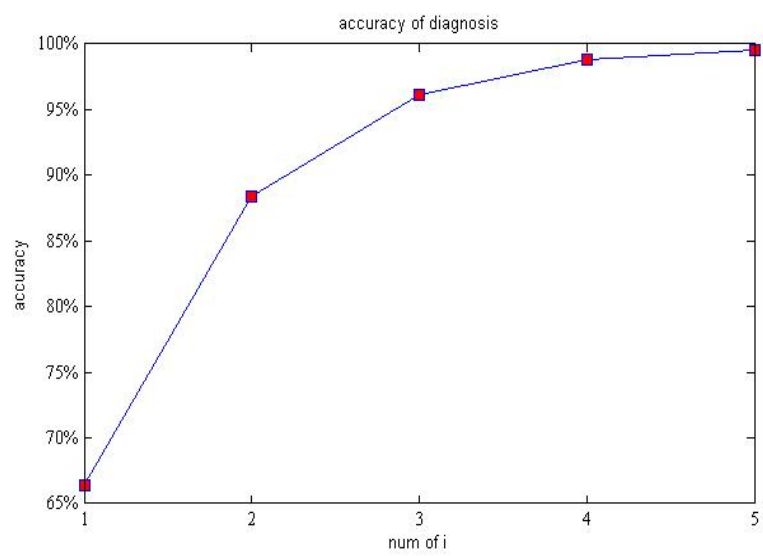

FIGURE IV. ACCURACY WITH THRESHOLD

At first, we wonder whether the threshold makes sense to the accuracy. Theoretically, the bigger of the threshold the higher of the accuracy. It is easy to comprehend that if the circulate number is reasonably high it can reach every possibility. Every fault detection we run the algorithm in 1000 times and analyze the result. Figure 4 verify our hypothesis. The bigger of the threshold the higher of the accuracy ratio. When the $\mathrm{i}$ is 5 , the accuracy is close to $100 \%$.

In order to make balance of the accuracy and calculation we set the threshold is 3 . Figure 5 demonstrates the accuracy with the network scale. As a whole, the accuracy decreases when the network scale increases. Before 900 nodes network, the 
accuracy is higher than $96 \%$, but decrease to $84 \%$ when the network nodes reaches to 1500 .

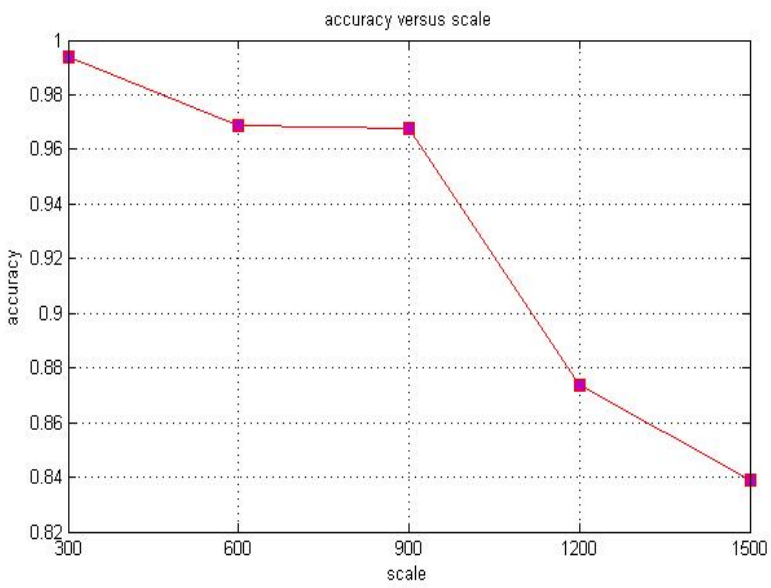

FIGURE V. ACCURACY WITH NETWORK SCALE

Figure 6 shows the accuracy in different conditions. The accuracy deceases when the network scale increases, however we can improve the accuracy by adding the threshold. For example, when the network size reaches at 1200 nodes if we still set the threshold 3 as before the accuracy falls down below to $90 \%$. Through increasing the threshold to 5, the accuracy can reach at more than $95 \%$. But in this way it will add the calculation work.

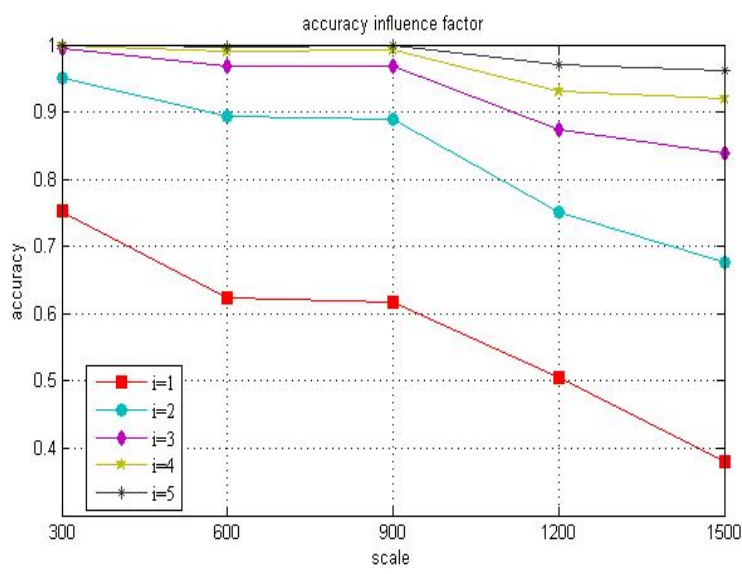

FIGURE VI. ACCURACY WITH INFLUNECE FACTOR

\section{CONCLUSION}

In this paper we introduce a new network model of space information network and give the detailed information about this model. Then under this circumstance we propose a selfdiagnosis algorithm based on finite-state machine. From the simulation result we can make a conclusion that the proposed algorithm performs well. However, we need to adjust the threshold when the network size changes. Next we will devote to adjusting the threshold automatically when the network size changes.

\section{ACKNOWLEDGMENT}

This work was supported by Major Research Plan of National Natural Science Foundation of China (No. 91438115); Jiangsu Province National Science Foundation (BK2011002); National Natural Science Foundation of China (No. 61371123).

\section{REFERENCES}

[1] Kun Dai, Changming Zhu, "A New Architecture Design of Space Information Networks", 2012 Sixth International Conference on Internet Computing for Science and Engineering.

[2] Wang K., Zhao Z.W., and Yao L., "An Agile Reconfigurable Key Distribution Scheme in Space Information Network", 2007 Second IEEE Conference on Industrial Electronics and Applications.

[3] Chunxiao Jiang, Xuexia Wang, Jian Wang, Hsiao-Hwa Chen, and Yong Ren, "Security in Space Information Networks", IEEE Communications Magazine, August 2015.

[4] Ren Fang, Fan Jiulun, "An adaptive distributed certificate management scheme for space information network", The Institution of Engineering and Technology 2013, Vol. 7, Iss. 4, pp. 318-326.

[5] Wanpeng, Wangruijun, Huangwei, "Analysis of the Performance of TCP and Its Extension Protocol for Space Communication," Journal of Spacecraft TT\&C Technolog, 2010, pp. 11-16.

[6] Liu jun, Han Huan, Geng Rong, Zheng zhong, Li Shao Hua, "Route Maintenance Strategy Based on the Packet Lost Distinguish for Space Information Networks", 2012 International Conference on Computer Science and Service System.

[7] Ning Ye, Zhiliang Zhu, Jun Liu, Jiping Shi, "Distributed Cluster-based Fault-tolerant Topology Control for Space Information Networks", 2010 International Conference on Cyber-Enabled Distributed Computing and Knowledge Discovery.

[8] Ning Ye, Zhiliang Zhu, Jun Liu, Weiyan Ren, "A SELFMAINTENANCE CLUSTERING ALGORITHM BASED ON DECISION MODEL FOR SPACE INFORMATION NETWORKS", Proceedings of ICCTA2009.

[9] http://www.nasa.gov/sites/default/files/files/Small_Spacecraft_Technolo gy_State_of_the_Art_2014.pdf.

[10] Kebin Liu, Qiang Ma, Xibin Zhao, Yunhao Liu, "Self-Diagnosis for Large Scale Wireless Sensor Networks", IEEE INFOCOM 2011, pp.1539-1547.

[11] Kebin Liu, Qiang Ma, Wei Gong, Xin Miao, Yunhao Liu, "SelfDiagnosis for Detecting System Failures in Large-Scale Wireless Sensor Networks", IEEE TRANSACTIONS ON WIRELESS COMMUNICATIONS, VOL.13, NO.10, OCTOBER 2014.

[12] A. Durresi, D. Dash, B. L. Anderson, R. Kannan, S. Kota, R. Jain, "Routing of Real-time Traffic in a Transformational Communications Architecture", 2004 IEEE Aerospace Conference Proceedings.

[13] Kimura K., Inagaki K. Double layered inclined orbit constellation for advanced satellite communications network[J]. IEICE Transaction on Communication, 1997, 8(1): 93-102.

[14] Fraire, J.A., Finochietto, J.M., "Design challenges in contact plans for disruption-tolerant satellite networks," IEEE Communications Magazine, Vol. 53 , No. 5, pp. 163-169, May 2015. 